\title{
Hyperchloremic Metabolic Acidosis with Hyperglycemic Hyperosmolar Syndrome after Robot-Assisted Radical Cystoprostatectomy with Ileal Conduit Urinary Diversion: A Case Report
}

\author{
Shugo Yajima Yasukazu Nakanishi Shunya Matsumoto Naoya Ookubo \\ Kenji Tanabe Hitoshi Masuda \\ National Cancer Center Hospital East, Chiba, Japan
}

\section{Keywords}

Hyperglycemic hyperosmolar syndrome $\cdot$ Hyperchloremic metabolic acidosis $\cdot$ Radical cystoprostatectomy $\cdot$ lleal conduit

\begin{abstract}
Hyperchloremic metabolic acidosis can be a problem in urinary diversion using the ileum. A 73-year-old Japanese male was hospitalized in emergency due to anorexia and malaise 3 weeks after being discharged from the hospital after getting robot-assisted radical cystoprostatectomy and intracorporeal ileal conduit urinary diversion. The blood analysis revealed metabolic acidosis, elevated chloride ions, and marked hyperglycemia: he was diagnosed with hyperchloremic metabolic acidosis and hyperglycemic hyperosmolar syndrome. We started administering insulin and large amounts of fluid replacement; besides, we inserted a largediameter open tip catheter into the ileal conduit in hopes of inhibiting urine reabsorption in the intestinal mucosa. His general condition gradually improved, and he was discharged 10 days after his hospitalization.
\end{abstract}

\section{Introduction}

Radical cystectomy with urinary tract reconstruction is the standard treatment for invasive bladder cancer; for urinary diversion, the ileal conduit has been commonly employed for many years. Although there have been frequently reported both hyperchloremic metabolic acidosis 
Fig. 1. CT of the abdomen showed no urine retention in the ileal conduit (a: white arrow); the IVC was collapsed (b: white circle), and intravascular dehydration was suspected. IVC, inferior vena cava.

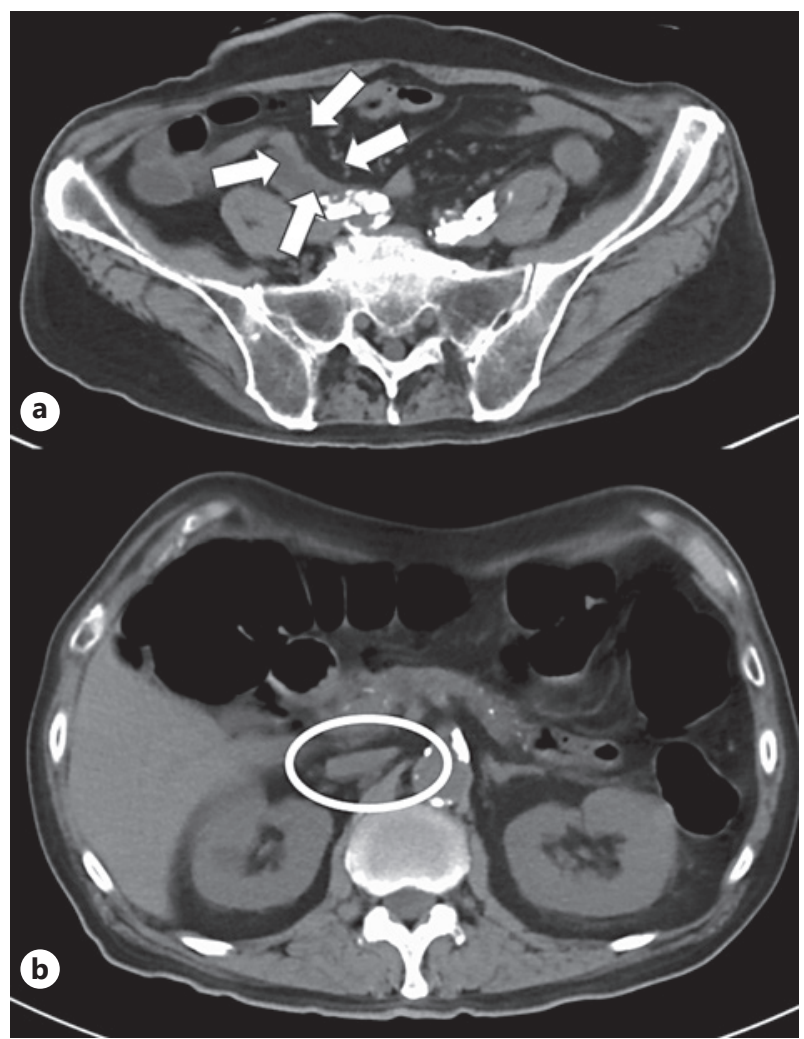

and disturbances in electrolyte metabolism in neobladder urinary diversion using the ileum, cases in ileal conduits have not been reported as often [1]. We herein report the case of a 73-year-old male diagnosed with hyperchloremic metabolic acidosis concomitant hyperglycemic hyperosmolar syndrome after robot-assisted radical cystoprostatectomy and intracorporeal ileal conduit urinary diversion.

\section{Case Report/Case Presentation}

A 73-year-old Japanese male was diagnosed with muscle-invasive bladder cancer: after 4 courses of neoadjuvant chemotherapy with cisplatin and gemcitabine, he underwent a robot-assisted laparoscopic radical cystoprostatectomy with intracorporeal ileal conduit urinary diversion using a Da Vinci Xi surgical system (Intuitive Surgical Inc., Sunnyvale, CA, USA). The length of the ileal conduit was approximately $20 \mathrm{~cm}$. The patient was released from the hospital 19 days after surgery with no major complications (Clavien-Dindo grade 0).

Three weeks after his discharge from the hospital, he presented to our outpatient clinic with complaints of anorexia and malaise. After his discharge from the hospital, he did not adhere to any dietary restrictions and consumed sugary drinks and alcohol frequently, but did not abuse any other drugs. The arterial blood gas analysis showed metabolic acidosis ( $\mathrm{pH}$ 7.19, bicarbonate $11.0 \mathrm{mEq} / \mathrm{L}$, base excess $-17.2 \mathrm{mEq} / \mathrm{L}$ ), and blood analysis revealed elevated chloride ions (115 mEq/L), marked hyperglycemia (1,095 mg/dL), and elevated plasma effective osmolarity (363 m0sm/L). The anion gap was normal, and no urine ketones were detected. CT of the chest-abdomen-pelvis showed no obvious findings other than a collapsed inferior vena cava and no urine retention inside the ileal conduit (shown in Fig. 1). 
Table 1. The data of blood analysis of the patient on admission

\begin{tabular}{ll}
\hline Laboratory test & $p$ value \\
\hline Arterial blood gas & \\
$\mathrm{pH}$ & 7.19 \\
$\mathrm{pCO}_{2}, \mathrm{~mm} \mathrm{Hg}$ & 28 \\
$\mathrm{pO}_{2}, \mathrm{~mm} \mathrm{Hg}$ & 98 \\
Base excess, mEq/L & -17.5 \\
Na, mEq/L & 135 \\
K, mEq/L & 5 \\
Cl, mEq/L & 115 \\
Bicarbonate, mEq/L & 11 \\
Blood analysis & \\
WBCs/ $\mu \mathrm{L}$ & 13,100 \\
CRP, mg/dL & 16.17 \\
Hemoglobin, g/dL & 12.1 \\
Creatinine, mg/dL & 1.54 \\
BUN, mg/dL & 90 \\
Anion gap & 10 \\
Glucose, mg/dL & $1-095$ \\
Posm, mOsm/L & 363 \\
Urinalysis & \\
RBCs/HPF & \\
WBCs/HPF & \\
Urine ketones & \\
\hline BUN, blood urea nitrogen; CRP, C-reactive protein; \\
\hline
\end{tabular}

We admitted him to the hospital as an emergency and started large amounts of fluid replacement. The data of his blood analysis on admission are shown in Table 1 . The glycated hemoglobin of this patient was $8.7 \%$ which was $6.7 \% 2$ months before the surgery.

Although the blood glucose level was gradually corrected by insulin administration, chloride ions and $\mathrm{pH}$ were not well controlled as of the day after admission (shown in Fig. 2). We continued to administer insulin and large amounts of fluid replacement; besides, we inserted a large-diameter open tip catheter (24 French scale) into the ileal conduit to inhibit urine reabsorption in the intestinal mucosa. The value of $\mathrm{pH}$ and chloride ions gradually improved (shown in Fig. 2). Eight days after admission, we removed the open tip catheter from the ileal conduit, and the patient was discharged from the hospital 10 days after admission.

\section{Discussion/Conclusion}

Diabetic ketoacidosis was suspected initially due to hyperglycemia and acidosis in the patient with poorly controlled type 2 diabetes mellitus, but it was ruled out based on the result of other laboratory data such as urine ketone and anion gap. We finally diag- 


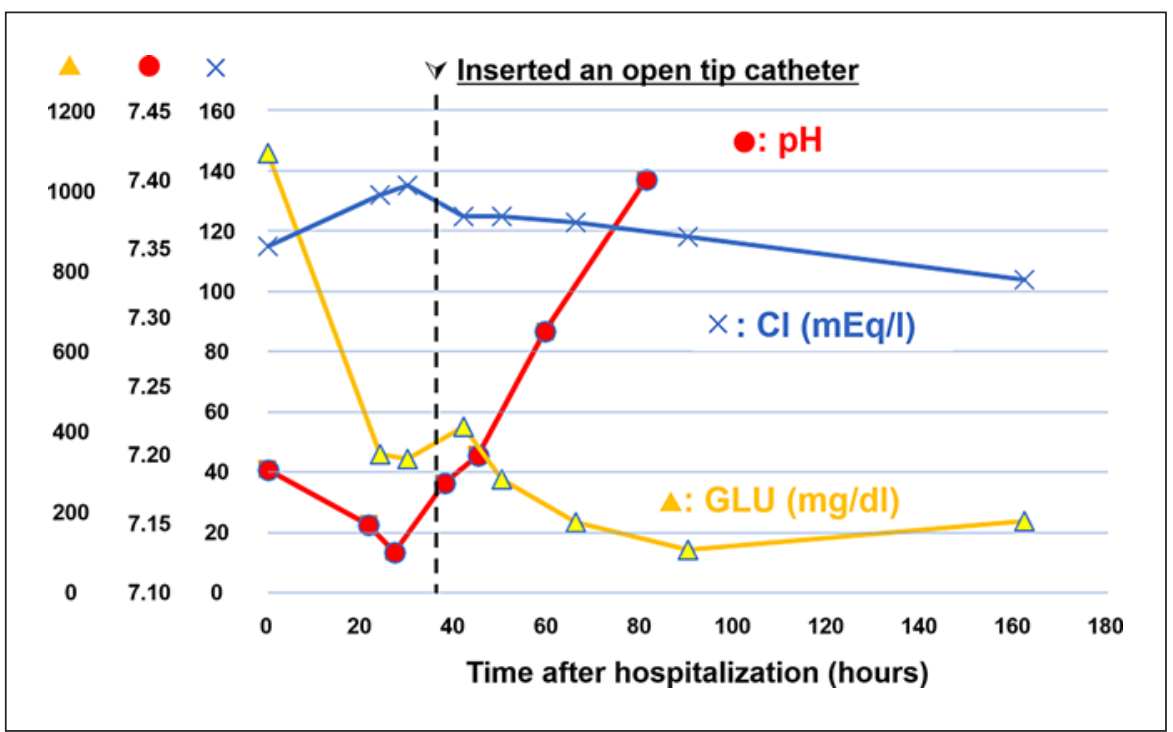

Fig. 2. Longitudinal trends in blood glucose, chloride ions, and $\mathrm{pH}$ of the patient after hospitalization.

nosed him with HHS because of a significant hyperglycemia of 1,095 mg/dL and a high plasma effective osmolality of $363 \mathrm{mOsm} / \mathrm{L}$. According to the recommendation of the American Diabetic Association and current international guideline, HHS is defined as plasma glucose level $>600 \mathrm{mg} / \mathrm{dL}$, plasma effective osmolarity $>320 \mathrm{mOsm} / \mathrm{L}$, and absence of significant ketoacidosis [2]. The pathogenesis of HHS in this patient is thought to have been triggered by an infection such as acute pyelonephritis in addition to the diabetes mellitus. Since early mortality of HHS was reported to be 16\% [3], early diagnosis is crucial for the patients.

On the other hand, hyperchloremic metabolic acidosis is a well-known complication after urinary diversion using the ileum [4]. In addition to HHS, this patient may have been in a state of hyperchloremic metabolic acidosis: acidosis is not usually seen in HHS [2, 3], but the $\mathrm{pH}$ of this patient may have decreased due to the concomitant hyperchloremic metabolic acidosis.

Concerning metabolic acidosis in patients undergoing a urinary diversion, the solute carrier family 26 member 3 (SLC26A3) was reported to play an important role in the etiology [5]. The protein encoded by SLC26A3 is a transmembrane glycoprotein that transports chloride ions across the cell membrane in exchange for bicarbonate ions; it is localized to the mucosa of the ileal epithelium, and contact with urine causes reabsorption of chloride ions and secretion of bicarbonate ions in the ileal epithelium, resulting in bicarbonate losing metabolic acidosis [5].

The large surface area of the intestine and the long contact time between the urine and the intestinal mucosa contribute to the development of metabolic complications [4], and therefore ileal conduits which have a shorter contact time with the urine and a smaller surface area of the intestine may be less prone to metabolic acidosis than a neobladder. However, in this report, the state of HHS causes an osmotic diuresis, and the patient became dehydrated; thus, the urine volume decreased and the velocity of urine passage in the ileal conduit was decreased, which accelerated the reabsorption of chloride ions and led to hyperchloremic metabolic acidosis. Because of the risk of increasing the plasma osmolality, we did not give an intravenous infusion of sodium bicarbonate, preferring to correct the hyperglycemia first.

\section{Karger'}


We placed a large-diameter open tip catheter in the ileal conduit in hopes of inhibiting the reabsorption of chloride ions from urine in the ileal conduit: this might have contributed to the rapid correction of $\mathrm{pH}$. However, there is a lack of scientific data to support this inference. If we had quantitatively measured the chloride ions in the urine before and after insertion of the open tip catheter, we might have been able to provide scientific evidence for our inference.

We report, for the first time, a case of hyperchloremic metabolic acidosis with HHS after robot-assisted radical cystoprostatectomy with ileal conduit urinary diversion. In the management of patients with underlying diabetes mellitus who have undergone urinary diversion, when hyperglycemia and acidosis are observed at the same time, it is necessary to keep in mind that HHS associated with urinary tract infection and hyperchloremic-metabolic acidosis may occur simultaneously; in these patients, postoperative dietary counselling, such as not abusing sugary drinks and alcohol, is also important.

\section{Acknowledgments}

The authors gratefully acknowledge the members of their department.

\section{Statement of Ethics}

Ethical approval to report this case was obtained from the National Cancer Center Institutional Review Board (Reference No. 2018-159). Written informed consent was obtained from the patient for publication of this case report and any accompanying images.

\section{Conflict of Interest Statement}

The authors have no conflicts of interest to declare.

\section{Funding Sources}

All authors have no funding sources to declare.

\section{Author Contributions}

Shugo Yajima wrote the first draft of the manuscript. Yasukazu Nakanishi revised the manuscript. All authors reviewed and edited the manuscript and approved the final version of the manuscript.

\section{Data Availability Statement}

Clinical data such as blood data and imaging data of the patients in the reported cases are kept by the authors. They can be disclosed if requested.

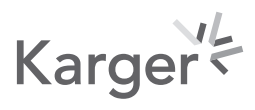




\section{References}

1 Cho A, Lee SM, Noh JW, Choi DK, Lee Y, Cho ST, et al. Acid-base disorders after orthotopic bladder replacement: comparison of an ileal neobladder and an ileal conduit. Ren Fail. 2017;39(1):379-84.

2 Adeyinka A, Kondamudi NP. Hyperosmolar hyperglycemic nonketotic coma. Treasure Island (FL): StatPearls Publishing CopyrightC 2021StatPearls Publishing LLC.; 2021.

3 Fadini GP, de Kreutzenberg SV, Rigato M, Brocco S, Marchesan M, Tiengo A, et al. Characteristics and outcomes of the hyperglycemic hyperosmolar non-ketotic syndrome in a cohort of 51 consecutive cases at a single center. Diabetes Res Clin Pract. 2011;94(2):172-9.

4 Vasdev N, Moon A, Thorpe AC. Metabolic complications of urinary intestinal diversion. Indian J Urol. 2013; 29(4):310-5.

5 McDougal WS. Metabolic complications of urinary intestinal diversion. J Urol. 1992;147(5):1199-208. 